\title{
AVALIAÇÃO HEMATOLOGICA DE CAPRINOS EXÓTICOS E NATIVOS NO SEMI-ÁRIDO PARAIBANO ${ }^{1}$
}

\author{
Hematological evaluation of exotic and native goats in Paraiba's Semi-arid
}

\author{
Elisângela Maria Nunes da Silva², Bonifácio Benício de Souza³, Gustavo de Assis Silva, Marcílio Fontes Cézar, \\ Marta Maria Soares Freitas ${ }^{5}$, Talicia Maria Alves Benício ${ }^{6}$
}

\begin{abstract}
RESUMO
O presente experimento foi conduzido na Estação Experimental de Pendência, pertencente à Empresa Estadual de Pesquisa Agropecuária da Paraíba S.A. (EMEPA), localizada no município de Soledade -PB, na microrregião do Curimataú Ocidental, Semi-árido da Paraíba. Objetivou-se com este trabalho realizar uma avaliação hematológica de caprinos exóticos e nativos criados no Semi-árido paraibano. Foram utilizadas 40 fêmeas das raças Boer, Savana, Anglo-Nubiana e Moxotó, sendo 10 de cada raça, com idade média de cinco meses, distribuídos num delineamento inteiramente casualizado, com 10 repetições, criadas em sistema semi-intensivo. Foram registradas as variáveis ambientais na sombra e no sol às 9:00 e às 15:00 horas, com auxílio de termômetros de máxima e mínima temperaturas, bulbo seco e bulbo úmido e de globo negro, e determinado o índice de temperatura do globo negro e umidade (ITGU), na sombra e no sol. Para a realização da hematimetria e leucometria foram coletadas amostras de $5 \mathrm{~mL}$ de sangue de cada animal, em tubo à vácuo com anticoagulante. A análise de variância revelou efeito de raça para os seguintes parâmetros hematológicos: eritrócitos, hematócrito e hemoglobina. Para o número de eritrócitos houve diferença significativa entre as raças Savana, Anglo-Nubiana e Moxotó, mas a raça Boer não diferiu em relação às raças Savana e Anglo-Nubiana. Houve efeito significativo para a variável leucócitos, tendo as raças Savana e Moxotó diferido das raças Boer e Anglo-Nubiana. Embora fatores como raça e ambiente influenciem sobre os parâmetros hematológicos, todas as raças apresentaram médias dentro dos limites normais para a espécie, demonstrando estarem bem adaptadas às condições climáticas do semi-árido.
\end{abstract}

Termos para indexação: Hemograma, adaptação, ambiente, raças.

\section{ABSTRACT}

The present experiment was carried out in the Experimental Station of Dispute, belonging to the State Company of Agricultural Research of Paraíba S.A. (EMEPA), located in the municipal district of Soledade -PB, in Curimataú Westerner small area, Semi-arid of Paraíba. One aimed with this, to accomplish an hematological evaluation of exotic and native goats servants in the semi-arid Paraiba. 40 females of the breed Boer, Savanna, Anglo-Nubiana and Moxotó, were used being, 10 of each breed, with medium age of five months, alloted in completely radomized design with 10 repetitions, grown in semi-intensive system. Environmental variables were registered in the shadow and sun at 9:00 and 15:00 o'clock with the aid of thermometers of maxim and low temperatures, dry bulb and humid bulb and black globe one. For the accomplishment of the hematimetria and leucometria, samples of $5 \mathrm{~mL}$ of blood of each animal were collected in vacuous tubes with anticoagulant. The variance analysis revealed breed effect for these hematological parameters: erythrocyte, hematocrit and hemoglobin. For the erythrocytes number there was significant difference among the breeds Savana, Anglo-Nubiana and Moxotó, but the Boer breed didn't differ in relation to the breeds Savana and Anglo-Nubiana. There was significant effect for the variable leukocyte that presented the breeds Savana e Moxotó differing from the breeds Boer e Anglo-Nubiana. Although factors with breed and atmosphere influence on the hematological parameters, all of the breed presented averages inside the normal limits for the species, demonstrating to be well adapted to the climatic conditions of the semi-arid.

Index terms: Hemogram, adaptability environment, breed.

(Recebido em 27 de junho de 2006 e aprovado em 25 de abril de 2007)

\footnotetext{
${ }_{1}^{1}$ Pesquisa financiada pelo CNPq/CAPES/UFCG/PATOS-PB.

${ }^{2}$ Mestre em Medicina Veterinária - Unidade Acadêmica de Medicina Veterinária/UAMV - Universidade Federal de Campina Grande/UFCG - Campus de Patos - Cx. P. 64 - 58700-970 - Patos, PB - elisangelamns@yahoo.com.br

${ }^{3}$ Doutores, Professores Adjunto - Unidade Acadêmica de Medicina Veterinária/UAMV - Universidade Federal de Campina Grande/UFCG - Campus de Patos - Cx. P. 64 - 58700-970 - Patos, PB - bonif@cstr.ufcg.edu.br; mfcezar@gmail.com

${ }^{4}$ Mestre em Medicina Veterinária - Instituto de Pesquisa Agropecuária de Pernambuco/IPA -Avenida Conselheiro João Alfredo, 56, sala 1 - 55330-000 - Bom Conselho, PE - gugavet2000@yahoo.com.br

${ }^{5}$ Médica Veterinária - Unidade Acadêmica de Medicina Veterinária/UAMV - Universidade Federal de Campina Grande/UFCG - Campus de Patos - CX. P. 64 - 58700-970 - Patos, PB - medvet.marta@gmail.com

${ }^{6}$ Mestranda - Unidade Acadêmica de Medicina Veterinária/UAMV - Programa de Pós-Graduação em Medicina Veterinária em Ruminantes e Eqüídeos/ PPGVM - Universidade Federal de Campina Grande/UFCG - Campus de Patos - Cx. P. 64 - 58700-970 - Patos, PB - taliciabenicio@yahoo.com.br
} 


\section{INTRODUÇÃO}

A caprinocultura na região Nordeste do Brasil desempenha grande destaque sócio-econômico para as populações rurais de baixa renda, por servir como fonte de proteína animal na alimentação humana, e também como fator de fixação do homem ao campo (BARROS et al., 1998). A criação de caprinos, conduzida em sua maior parte de forma extensiva vem se apresentando com potencial aquém do real, implicando em baixa produtividade, baixa taxa de desfrute e, consequientemente, insatisfatórios resultados econômicos.

Nos últimos anos, melhorias têm sido conseguidas por meio da adoção de tecnologias e pesquisas avançadas, junto a práticas de manejo racional e programas de melhoramento genético dos plantéis (NOGUEIRA FILHO, 2003). Contudo, muita atenção deve ser dada, quando animais de outras regiões são utilizados em programas de melhoramento, uma vez que fatores individuais como: raça, sexo, idade, alimentação e manejo (LINDSTROM, 1982), além das variáveis ambientais como: clima, altitude, umidade relativa do ar e temperatura ambiente podem apresentar evidentes variações dos elementos constituintes do hemograma, interferindo na adaptabilidade dos animais (VIANA et al., 2002).

No entanto, apesar da variedade de fatores que podem interferir nos valores hematológicos dos animais, muito pouco se tem disponível na literatura, sobre os efeitos deles, nas raças criadas na região Nordeste, sendo necessários mais estudos inerentes ao tema, de forma a esclarecer como esses fatores influenciam realmente no perfil hematológico.

Segundo Paes et al. (2000), o hemograma é um exame complementar que fornece ao profissional da área de produção animal várias informações sobre o estado de saúde dos animais e também serve como indicador de estresse térmico. Assim sendo, diversos pesquisadores, das mais variadas regiões do mundo, têm se preocupado em estabelecer valores referenciais para o número de hemácias e outros constituintes sanguíneos. Por esse motivo, os parâmetros hematológicos devem ser preferencialmente regionais (BIRGEL JÚNIOR et al., 2001; JAIN, 1993). Silveira (1988) descreveu o hematócrito como sendo uma estimativa da massa de eritrócitos em relação ao volume sanguíneo. De acordo com Nunes et al. (2002), quanto maior a solicitação física do animal maior será o valor do hematócrito por causa da perda de líquidos através da forma evaporativa.

Lee et al. (1974), relataram que o hematócrito pode estar diminuído em função de anemias, hemólise, prenhez avançada e um estresse térmico por longa duração pode reduzir o número de hemácias, o teor de hemoglobina e a contagem global de leucócitos, em razão de um processo de hemodiluição. Segundo Schimidt-Nielsen (1996), a função da hemoglobina consiste no transporte de oxigênio dos pulmões para os diferentes tecidos e durante o esforço físico a liberação do oxigênio se processa de forma mais rápida, contribuindo para a elevação na taxa de consumo de oxigênio e conseqüentemente aumento do valor da hemoglobina. Em virtude dessas variações, o estudo hematológico de raças exóticas e nativas, na região Semiárida, é de fundamental importância para a determinação de genótipos mais adaptados à condição ambiental específica dessa região. Objetivou-se com este trabalho realizar uma avaliação hematológica de caprinos exóticos e nativos criados no Semi-árido paraibano.

\section{MATERIAL E MÉTODOS}

O experimento foi conduzido na Estação Experimental de Pendência, pertencente à Empresa Estadual de Pesquisa Agropecuária da Paraíba S.A. (EMEPA), localizada no município de Soledade-PB, na microrregião do Curimataú Ocidental, Semi-árido Paraibano, situada à $7^{\circ} 8^{\prime} 18^{\prime}$ "S e $36^{\circ} 27^{\prime}$ 2"'W. Gr., a uma altitude média de $534 \mathrm{~m}$. Foram utilizadas 40 fêmeas, sendo 10 de cada raça: Boer, Savana, Anglo-Nubiana e Moxotó, com idade média de cinco meses, distribuídos num delineamento inteiramente casualizado, com quatro tratamentos, constituídos pelas raças: Boer, Savana, Anglo-Nubiana e Moxotó, com 10 repetições.

Os animais foram mantidos em regime semiintensivo, tendo como base alimentar a vegetação nativa (caatinga) e como suplementação receberam uma ração completa. A ração era composta por $29 \%$ de feno de Tifton (Cynodon spp.), $25 \%$ de farelo de soja, $15 \%$ de farelo de trigo, $30 \%$ de milho triturado e $1 \%$ de mistura mineral; a qual foi oferecida duas vezes ao dia, às 7:00 horas e às 13:00 horas, num total de 300 gramas/cabeça/dia.

Durante o período experimental foram registradas as variáveis ambientais na sombra e no sol às 8:00 e às 15:00 horas com auxílio de termômetros de: máxima e mínima temperaturas, do bulbo seco e bulbo úmido, termômetros de globo negro e determinado o índice de temperatura do globo negro e umidade (ITGU), na sombra e no sol, de acordo com a fórmula: ITGU $=$ Tgn $+0,36($ Tpo $)+41,5$ (BUFFINGTON et al., 1981).

Para a realização da hematimetria e leucometria foram coletadas amostras de $5 \mathrm{~mL}$ de sangue de cada animal em tubo à vácuo, com anticoagulante etileno-diamino- 
tetracetato de sódio (EDTA) a 10\%. As amostras de sangue foram mantidas em isopor com gelo até sua chegada ao Laboratório de Patologia Clínica do Hospital Veterinário do Centro de Saúde e Tecnologia Rural da Universidade Federal de Campina Grande (CSTR-UFCG). As contagens de eritrócitos (Er) e leucócitos totais foram realizadas em câmara do tipo Neubauer modificada e, para tanto, a diluição das células foi feita utilizando-se pipeta semiautomática de 20 microlitros conforme preconizada por Vallada (1999). Para determinação do hematócrito (Ht), utilizou-se à técnica do microhematócrito por 15 minutos, conforme descrito por Ayres (1994), na qual utilizaram-se tubos capilares homogêneos de 75 milímetros de comprimento por um milímetro de diâmetro. A determinação do teor de hemoglobina $(\mathrm{Hb})$ no sangue foi realizada pelo método da cianometahemoglobina, conforme técnica descrita por Mello (2001), utilizando o analisador bioquímico automático, BIOPLUS 2000, com auxílio de Kit comercial próprio para dosagem de hemoglobina, LABTEST DIAGNÓSTIC.

Os índices hematimétricos absolutos: volume globular médio (VGM) e concentração de hemoglobina globular média (CHGM) foram obtidos a partir da contagem do número de Er, do $\mathrm{Ht}$ e do teor de $\mathrm{Hb}$, segundo Ferreira Neto \& Viana (1977). A contagem diferencial de leucócitos foi realizada utilizando sangue "in natura" através da confecção de dois esfregaços sanguíneos, corados com corante de Rosenfeld e examinados ao microscópio com objetiva de imersão a óleo (100x), segundo técnica padronizada por Birgel (1982). Em cada esfregaço sanguíneo foram diferenciados 100 leucócitos, classificados de acordo com suas características morfológicas e tintoriais em neutrófilos com núcleo segmentado, eosinófilos, basófilos, linfócitos e monócitos. Os dados obtidos foram analisados através do Programa de Análises Estatísticas e Genéticas -SAEG (UFV, 1993) e as médias foram comparadas pelo teste de Tukey, a 5\% de probabilidade.

\section{RESULTADOS E DISCUSSÃO}

As médias das variáveis ambientais e do ITGU, observadas durante o experimento foram: temperatura de bulbo seco $29,50{ }^{\circ} \mathrm{C}$, temperatura de bulbo úmido, $21,51^{\circ} \mathrm{C}$; temperatura de globo negro na sombra $32,16{ }^{\circ} \mathrm{C}$; temperatura de globo negro no sol $44,27{ }^{\circ} \mathrm{C}$; umidade relativa do ar 50,08 \%; índice de temperatura do globo negro e umidade na sombra $80,11^{\circ} \mathrm{C}$; e índice de temperatura do globo negro e umidade no sol $92,17^{\circ} \mathrm{C}$.

A análise de variância revelou efeito de raça $(\mathrm{P}<0,05)$ sobre os parâmetros hematológicos: eritrócitos, hematócrito e hemoglobina, conforme observa-se na Tabela 1.

Com relação ao número de eritrócitos houve diferença $(\mathrm{P}<0,05)$ entre as raças, tendo a Moxotó apresentado a maior média e a Savana a menor, porém a raça Boer não diferiu das raças Savana e Anglo-Nubiana, Tabela 1. A raça Moxotó seguida da raça Anglo-Nubiana apresentaram maior número de eritrócitos, podendo esta ser uma característica adquirida ao longo dos anos no processo de adaptação às condições climáticas da região, uma vez que essas duas raças estão presentes na região há muitos anos. Concordando com Lee et al. (1974) e Viana et al. (2002) quando descrevem sobre a influência de fatores ambientais sobre os parâmetros hematológicos. Segundo Swenson (1996) quanto maior o número de eritrócitos, maior a capacidade de oxigenação dos tecidos através da oxiemoglobina, já que durante a passagem dos eritrócitos pelos capilares pulmonares a hemoglobina combina com o oxigênio formando a oxiemoglobina, que ao atravessar os capilares sistêmicos, perde seu oxigênio para os tecidos. Contudo, todas as raças apresentaram valores para eritrócitos dentro da normalidade e dentro da média descrita por Ferreira Neto et al. (1982), Jain (1993), Oduye (1976) e Viana et al. (2003).

Os valores do hematócrito diferiram $(\mathrm{P}<0,05)$ para todas as raças (Tabela 1) tendo a Moxotó superado as

Tabela 1 - Valores médios do eritrograma de caprinos exóticos e nativos no semi-árido: eritrócitos (Er), hematócrito (Ht), hemoglobina (Hb), volume globular médio (VGM) e concentração de hemoglobina globular média (CHGM).

\begin{tabular}{lccccc}
\hline & \multicolumn{5}{c}{ Parâmetros hematológicos } \\
\cline { 2 - 6 } Raças & $\mathrm{Er}\left(\mathrm{mm}^{3}\right)$ & $\mathrm{Ht}(\%)$ & $\mathrm{Hb}(\mathrm{g} / \mathrm{dl})$ & $\mathrm{VGM}\left(\mu^{3}\right)$ & $\mathrm{CHGM}(\%)$ \\
\hline Bôer & $14.934,00 \mathrm{BC}$ & $25,50 \mathrm{C}$ & $8,38 \mathrm{C}$ & $17,24 \mathrm{~A}$ & $32,86 \mathrm{~A}$ \\
Savana & $13.689,00 \mathrm{C}$ & $23,20 \mathrm{D}$ & $7,73 \mathrm{C}$ & $16,97 \mathrm{~A}$ & $33,31 \mathrm{~A}$ \\
Anglo-Nubiana & $16.153,00 \mathrm{~B}$ & $27,90 \mathrm{~B}$ & $9,38 \mathrm{~B}$ & $17,30 \mathrm{~A}$ & $33,60 \mathrm{~A}$ \\
Moxotó & $17.846,00 \mathrm{~A}$ & $30,20 \mathrm{~A}$ & $10,15 \mathrm{~A}$ & $16,97 \mathrm{~A}$ & $33,60 \mathrm{~A}$ \\
\hline CV $(\%)$ & 8,65 & 6,44 & 6,93 & 7,26 & 2,79 \\
\hline
\end{tabular}

Médias seguidas de letras diferentes na coluna diferem estatisticamente entre si $(\mathrm{P}<0,05)$ pelo teste de Tukey. 
demais. Para Silva \& Costa (1982) a variação no hematócrito está relacionada com o fator raça. Segundo Bianca (1965) a variação do hematócrito depende da severidade da carga calórica imposta sobre o animal. Animais que sofrem com estresse prolongado tendem a apresentar redução do hematócrito (HERZ \& STEINHAUT, 1978). No entanto, os valores encontrados para o hematócrito encontram-se dentro da normalidade de acordo com Jain (1986), Souza (2003) e Viana et al. (2002) e discordam dos achados por Dantas (1984) quando descrevem não haver influência da temperatura ambiente sobre a redução do volume globular. Para hemoglobina não houve diferença significativa $(\mathrm{P}>0,05)$ entre as raças Boer e Savana, (Tabela 1) porém houve diferença $(\mathrm{P}<0,05)$ com relação às outras duas raças, tendo a raça Moxotó apresentado a maior média, discordando de Souza (2003), que não encontrou diferença significativa quando trabalhou com mestiços dos grupos genéticos Boer, Savana, Moxotó e Anglo-Nubiana, em condições de confinamento. Os valores médios encontrados para eritrócitos, hematócrito e hemoglobina apresentaram-se superiores para a raça Moxotó e esses estão de acordo com os encontrados por Unanian (1986) que, ao estudar os parâmetros sanguíneos de caprinos jovens de diversas raças no Semi-árido do Brasil, também observou diferença significativa entre as raças e valores superiores para a raça Moxotó.

Para o VGM e CHGM não foram encontradas diferenças significativas entre as raças; no entanto, as médias dessas variáveis se apresentaram dentro da normalidade, o VGM 16 a $25 \mathrm{~m}^{3}$ e CHGM 30 a $36 \%$ encontrados por Jain (1986) e Lopes (1996).

A análise de variância do leucograma revelou efeito $(\mathrm{P}<0,05)$ de raça para a variável leucócitos, porém, não foi observada para as outras variáveis, conforme observa-se na Tabela 2.
Houve diferença significativa $(\mathrm{P}<0,05)$ para a contagem total de leucócitos entre as raças Moxotó e Savana, tendo a Moxotó apresentado a maior média e a Savana a menor; com relação às raças Boer e AngloNubiana não foi observada diferença significativa $(\mathrm{P}>0,05)$ entre elas, resultados esses, que diferiram dos encontrados por Souza (2003), que não observou diferença significativa $(\mathrm{P}>0,05)$ para a variável leucócitos, ao avaliar o leucograma de caprinos mestiços dos mesmos grupos raciais, criados intensivamente. Para as variáveis: número absoluto de segmentados, linfócitos, eosinófilos e monócitos, não foi detectada diferença significativa ( $\mathrm{P}>0,05)$, entre os grupos raciais. Edjtehadi (1978) trabalhando com caprinos em regime extensivo, suplementados com concentrado, encontrou os seguintes resultados para leucócitos 14,45 $\pm 2,75\left(10^{3} \mathrm{~mm}^{3}\right)$. Ferreira Neto et al. (1982), avaliando o leucograma de caprinos criados em regime semi-intensivo e em confinamento não observaram diferença estatística entre os grupos e obtiveram os seguintes valores para leucócitos: de 9,91 a $24,34\left(10^{3} \mathrm{~mm}^{3}\right)$. Nesse trabalho os valores encontrados para leucócitos apresentaram dentro da normalidade e estão em concordância com os encontrados por Gautam (1965), Greenwood (1977) e Magnífico \& Rosa (1982) e acima dos encontrados Oduye (1976) e com os de Ferreira Neto et al. (1982).

É importante ressaltar que todos os índices encontrados nesse estudo mantiveram-se dentro da faixa de normalidade estabelecida para outras raças caprinas, verificando-se que as raças tanto exóticas como nativas, responderam satisfatoriamente às condições ambientais, mantendo os parâmetros hematológicos dentro dos padrões considerados normais para a espécie, sendo um indicativo de boa adaptação às condições do Semiárido.

Tabela 2 - Valores médios do leucograma de caprinos exóticos e nativos no semi-árido paraibano.

\begin{tabular}{llcccc}
\hline Raças & $\begin{array}{c}\text { Leucócitos } \\
\left(10^{3} \mathrm{~mm}^{3}\right)\end{array}$ & $\begin{array}{c}\text { Segmentados } \\
(\%)\end{array}$ & $\begin{array}{c}\text { Linfócitos } \\
(\%)\end{array}$ & $\begin{array}{c}\text { Eosinófilos } \\
(\%)\end{array}$ & $\begin{array}{c}\text { Monócitos } \\
(\%)\end{array}$ \\
\hline Boer & $12.046,00 \mathrm{AB}$ & $38,10 \mathrm{~A}$ & $61,00 \mathrm{~A}$ & $0,70 \mathrm{~A}$ & $0,10 \mathrm{~A}$ \\
Savana & $11.265,00 \mathrm{~B}$ & $37,00 \mathrm{~A}$ & $61,90 \mathrm{~A}$ & $0,90 \mathrm{~A}$ & $0,10 \mathrm{~A}$ \\
Anglo-Nubiana & $11.865,00 \mathrm{AB}$ & $38,00 \mathrm{~A}$ & $60,10 \mathrm{~A}$ & $1,70 \mathrm{~A}$ & $0,20 \mathrm{~A}$ \\
Moxotó & $14.264,00 \mathrm{~A}$ & $43,20 \mathrm{~A}$ & $55,00 \mathrm{~A}$ & $0,70 \mathrm{~A}$ & $0,60 \mathrm{~A}$ \\
\hline
\end{tabular}

Médias seguidas de letras diferentes na coluna diferem estatisticamente entre si $(\mathrm{P}<0,05)$, pelo teste de Tukey. 


\section{CONCLUSÕES}

Conclui-se que o fator raça exerceu influência sobre os parâmetros hematológicos: eritrócitos, hematócrito, hemoglobina e leucócitos, no entanto, os valores encontrados para cada raça não podem ser estabelecidos como absolutos, sem levar em consideração as condições de criação e as diferenças regionais.

Todas as raças estudadas apresentaram médias dentro dos limites normais para a espécie, demonstrando estarem bem adaptadas às condições climáticas do Semi-árido.

\section{REFERÊNCIAS BIBLIOGRÁFICAS}

AYRES, M. C. C. Eritrograma de Zebuínos (Bos indicus, Linnaeus, 1759) da raça Nelore, criados no Estado de São Paulo, influência dos fatores etários, sexual e do tipo racial. 1994. Dissertação (Mestrado) - Universidade de São Paulo, São Paulo, 1994.

BARROS, N. N. et al. Teste de desempenho reprodutivo de ovinos Santa Inês e Somalis Brasileira da Embrapa. Sobral: Empresa Brasileira de Pesquisa Agropecuária, 1998. (Boletim técnico, 36).

BIANCA, W. Reviews of the progress of dairy science. Journal Dairy Research, Cambridge, n. 32, p. 291-345, 1965.

BIRGEL, E. H. Hematologia clínica veterinária. In: BIRGEL, E. H.; BENESI, F. J. Patologia clínica veterinária. São Paulo: Sociedade Paulista de Medicina Veterinária, 1982. p. 2-34.

BIRGEL JÚNIOR, E. H. et al. Valores de referência do eritrograma de bovinos da raça Jersey criados no Estado de São Paulo. Arquivo Brasileiro de Medicina Veterinária e Zootecnia, Belo Horizonte, v. 53, n. 2, p. 164-171, 2001.

BUFFINGTON, D. E.; COLLAZO-AROCHO, A.; CANTON, G. H.; PITT, D. Black Globe-Humidity index (BGHI) as comfort equation for dairy cows. Transactions of the Asae, [S.l.], p. 711-713, 1981.

DANTAS, M. O. Relação entre temperatura ambiente, corporal, freqüiência de pulso e volume globular de caprinos (Capra hircus). 1984. 77 p. Dissertação (Mestrado) - Universidade Federal Rural de Pernambuco, Recife, 1984.

EDJTEHADI, H. Age associated changes in the blood pícture of the goat. Zentralb. Veterinarmed, Hamburg, v. 25, n. 3, p. 198-206, 1978.
FERREIRA NETO, J. M.; BIONDINI, J. B.; CARVALHO, M. M. Leucograma de caprinos confinados e em pastoreio semi-extensivo. Arquivos da Escola de Veterinária da UFMG, Belo Horizonte, v. 34, n. 2, p. 221-227, 1982.

FERREIRA NETO, J. M.; VIANA, E. S. Patologia clínica veterinária. Belo Horizonte: Rabelo, 1977. 279 p.

GAUTAM, O. P. Hematological norms in goats. Indian Journal Veterinary Science, New Delhi, v. 35, p. 173-177, 1965.

GREENWOOD, B. Hematology of the sheep and goat. In: ARCHER, L. K.; JEFFCOTT, L. B. Comparative clinical haematology. Oxford: Blackwell Scientific, 1977. p. 305.

HERZ, A.; STEINHAUT, D. The reaction of domestic animal to heat stress. Animal Research Development, [S.1.], n. 7, p. 7-38, 1978.

JAIN, N. C. Essentials of veterinary hematology. Philadelphia: Lea \& Febiger, 1993. 417 p.

JAIN, N. C. Schalm's veterinary hematology. 4. ed. Philadelphia: Lea \& Febinger, 1986. 1221 p.

LEE, J. A.; ROUSSEL, J. D.; BEATTY, J. F. Effect of temperature season on bovine adrenal cortical function, blood cell profile, and milk production. Journal of Dairy Science, Champaign, v. 59, n. 1, p. 104-108, 1974.

LINDSTROM, U. P. Sustâncias bioquímicas indicadoras en la cria animal. Revista Mundial de Zootecnia, [S.l.], v. 42, p. 35-38, 1982.

LOPES, S. T. A.; CUNHA, C. M. S.; BIONDO, A. W.; FA, L. C. Patologia clínica veterinária. Santa Maria: UFSM, 1996. 161 p.

MAGNÍFICO, P. F.; ROSA, S. D. M. Algunos valores hematológicos en animales clinicamente sanos esplotados en el estado Aragua: ovejas, cabras y equinos. Vet. Trop., [S.1.], v. 7, p. 59-75, 1982.

MELLO, M. T. Hemograma referencial de caprinos criados no Estado de Pernambuco: procedimentos clínicolaboratoriais e avaliação da influência dos fatores etário e sexual. 2001. 72 p. Dissertação (Mestrado em Medicina Veterinária - Clínica Médica) - Universidade Federal Rural de Pernambuco, Recife, 2001. 
NOGUEIRA FILHO, A. Ações de fomento do banco do nordeste e potencialidades da caprino-ovinocultura. In: SIMPÓSIO INTERNACIONAL SOBRE CAPRINOS E OVINOS DE CORTE, 2., 2003, João Pessoa. Anais... João Pessoa: Governo do Estado da Paraíba, 2003. p. 43-55.

NUNES, A. S. et al. Efeito de dois regimes de suplementação e dois sistemas de produção, nos constituintes sangüíneos de cabras Saanen durante a lactação. Revista Brasileira de Zootecnia, Viçosa, v. 31, n. 3, p. 1245-1250, 2002.

ODUYE, O. O. Hematological values of Nigerian goats and sheep. Tropical Animal Heath Production, Edimburgh, v. 8, n. 3, p. 131-136, 1976.

PAES, P. R.; BARIONI, G.; FONTEQUE, J. R. Comparação dos valores hematológicos entre caprinos fêmeas da raça Parda Alpina de diferentes faixas etárias. Veterinária Notícias, [S.1.], v. 6, n. 1, p. 43-49, 2000.

SCHIMIDT-NIELSEN, K. Fisiologia animal adaptação e meio ambiente. 5. ed. São Paulo: Santos, 1996. 546 p.

SILVA, M. U. D.; COSTA, C. A. F. Determinação de valores sanguíneos em caprinos jovens de diferentes raças. In: CONGRESSO BRASILEIRO DE MEDICINA VETERINÁRIA, 18., 1982, Balneário de Camboriú, Santa Catarina. Anais... Santa Catarina: Sociedade Brasileira de Medicina Veterinária, 1982. p. 233.

SILVEIRA, J. M. Patologia clínica veterinária: teoria e interpretação. Rio de Janeiro: Guanabara Koogan, 1988. 196 p.
SOUZA, E. D. Respostas fisiológicas de caprinos de diferentes grupos genéticos às condições do semi-árido nordestino. 2003. 83 f. Dissertação (Mestrado em Zootecnia) - Universidade Federal da Paraíba, Areia, 2003

SWENSON, M. J. Circulação sanguínea e sistema cardiovascular. In Dukes fisiologia dos animais domésticos. 11. ed. Rio de Janeiro: Guanabara, 1996. cap. 2, p. 13-34.

UNANIAN, M. M. Parâmetros sangüíneos em caprinos jovens da região semi-árida do Nordeste do Brasil. Pesquisa Agropecuária Brasileira, Brasília, v. 21, n. 3, p. 293-301, 1986.

UNIVERSIDADE FEDERAL DE VIÇOSA. SAEG - Sistema de Análises Estatísticas e Genéticas. Viçosa, 1997.

VALLADA, E. P. Manual de técnicas hematológicas. São Paulo: Atheneu, 1999. 423 p.

VIANA, R. B. et al. Influência da gestação e do puerpério sobre o leucograma de caprinos da raça Saanen, criados no Estado de São Paulo. Brazilian Journal Veterinary Research Animal Science, São Paulo, v. 39, n. 4, p. 196201, 2002.

VIANA, R. B. et al. Influência da gestação e do puerpério sobre o eritrograma de caprinos (Capra hircus) da raça Saanen, criados no Estado de São Paulo. Brazilian Journal Veterinary Research Animal Science, São Paulo, v. 40, n. 3, p. 178-184, 2003. 